\title{
VARICELLA ZOSTER MENINGITIS IN AN IMMUNIZED CHILD
}

A 5-year-old girl with varicella-zoster virus meningitis who had received the VZV vaccine 18 months previously is reported from the University of Chicago. She presented with a 5-day history of fever, headache, and a rash on the face and trunk, with diffuse papules, vesicles, and scabbed lesions. CSF showed 715 white cells with $97 \%$ lymphocytes, protein $133 \mathrm{mg} / \mathrm{dL}$, glucose $46 \mathrm{mg} / \mathrm{dL}$ (serum glucose $86 \mathrm{mg} / \mathrm{dL}$ ), and no bacteria on Gram-stain. CSF was positive for VZV by polymerase chain reaction. After a 10- day course of acyclovir, she made an uneventful recovery. A follow-up hearing test 6 weeks later revealed a right-sided sensorineural hearing loss, which had resolved when tested 2 years later. (Schwab J, Ryan M. Varicella zoster virus meningitis in a previously immunized child. Pediatrics August 2004;114:e273-e274). (Respond: Joel Schwab MD, 5839 S Maryland Ave, MC 3055, Chicago, IL 60637).

COMMENT. In an earlier report of VZV meningitis in a previously immunized child, there was evidence of seroconversion 4 weeks after immunization (Naruse et al. 1993), and the child had been exposed to a case of varicella 2 weeks before the illness. In the present case, there was no laboratory evidence of seroconversion and no known exposure to varicella. Although rarely reported, breakthrough cases of varicella with neurologic complications may occur in vaccinated children.

\section{VASCULAR DISORDERS}

\section{CEREBRAL SINUS THROMBOSIS AND PERIORBITAL BRUISING}

Two patients, females aged 24 and 25 years, with raised intracranial pressure and dural venous sinus thrombosis, who presented with periorbital bruising, are reported from Moorfields Eye Hospital, and the National Hospital for Neurology and Neurosurgery, London, UK. Patient 1 had a history of migraine, and spontaneous periorbital bruising was preceded by a 1-week history of severe generalized headache that awakened her at night and was associated with vomiting, photophobia, and neck stiffness. She had bilateral acute papilledema, and brain CT findings were consistent with a thrombosis of the superior sagittal sinus and a venous infarct in the right frontal lobe, confirmed by MRI and MR venography. Periorbital bruising resolved after treatment with heparin and warfarin. Patient 2 was 25 weeks pregnant when she presented with periorbital bruising, headache, neck stiffness, vomiting, and photophobia. She had a sixth nerve palsy and papilledema, and the MRI and MRV were normal. CSF had an opening pressure of $46 \mathrm{~cm}$, and normal constituents. Headache improved after lumbar puncture, and bruising resolved in the weeks following treatment with acetazolamide. Dural sinus thrombosis was considered likely. (Hadjikoutis S, Carroll C, Plant GT. Raised intracranial pressure presenting with spontaneous periorbital bruising: two case reports. J Neurol Neurosurg Psychiatry August 2004;75:1192-1193). (Respond: Dr GT Plant, The National Hospital for Neurology and Neurosurgery, Queen Square, London, WC1N 3BG, UK). 